\title{
Position Tracking and Fuzzy Logic
}

\author{
Mohd. Asim \\ MGM CoET Noida \\ Uttar Pradesh, India
}

\author{
Riya Malik \\ MGM CoET, Noida \\ Uttar Pradesh, India
}

\author{
Shagun Bhatt \\ MGM CoET, Noida \\ Uttar Pradesh, India
}

\begin{abstract}
In this paper, the problem of tracking the position of an object from a remote place is solved by applying the rules of fuzzy logic. The domain of image analysis and artificial intelligence has been integrated so as to track the position of the object and get results that are more precise. The object has been identified and then tracked until it has exceeded the range of a camera. Various techniques have been used at various levels of the entire process. Fuzzy rules have been made so as to accomplish the goal. A video has been first converted into frames and then using technique of background subtraction, the object has been identified and then tracked which is an optimal solution as well as precise.
\end{abstract}

Keywords : Fuzzy Logic, Position Tracking, Fuzzy Inference System.

\section{INTRODUCTION}

Position Tracking using Fuzzy Logic is the leading field in the world of research. Today lots of research is being done in the field of Tracking the position of a remote object. The need to track position of a person or an object can be due to various reasons and so are the procedures. Numerous number of procedures has been developed in order to fulfil this need. Technologies and software are also present but it is our will in order to use which way of solving the problem of position tracking.

Position Tracking: In this, first a video has been captured located in an area and then that video has been converted into frames using functions of matlab. The whole process has been divided into three steps:

First is the step of object identification in which the technique of background subtraction has been used. In this, first the intensity values of all the pixels are calculated and by background modelling the pixels intensities are arranged. A threshold value is set above which all the values are considered to be of the object of interest and rest are considered to be the pixels of the background. In this way, the object is identified and background subtraction is performed in order to get the desired object. Second step is to correctly classify the different objects which have been identified in the background according to the rules. The system is made to learn some rules through the process of supervised learning. The third and the final step is to precisely track the position of the object. This is the most crucial step and involves very close analysis of the step to step movement of the object. The whole procedure of doing this process is being explained in this paper.

\section{LITRATURE SURVEY}

Position Tracking using fuzzy logic has many implementations among which the first that we came across was a Robot Soccer System, consisting of Robots and a Soccer ball. In this the Robots are divided into two teams and they both are playing a match. All the robots in this game are designed in such a way that each one of them will initially detect the position of the ball and then try to fetch a goal. The ball will be detected by a camera placed on the head of the robot. This camera once captures the image of the ball then detects the angle between opponent player ball and if there is no interruption then the ball is kicked a goal is fetched [1].

The second paper that we studied was on how can vehicles track an obstacle in front if it and then automatically takes decision based on the situation. This paper tells us about an Automatic Breaking System used in the cars which can detect the obstacles or object present in front of it. This was done using the condition that is the object present in front makes an angle less than 60 degrees then the object is ignored and if it I more than 60 degrees then the automatic breaking system gets $\mathrm{ON}$ and breaks are applied. This paper tells us how can objects be detected in front and then according to the situation occurring the decision is taken[2].

The methodology of Colour Based Segmentation is another such technique used in the field of position tracking this is applied by recognising the colour of the target and then throughout the process matching it and keeping a track of it, so as to track the displacement of an objectfrom initial position to final one. This method is simple yet efficient as only the colour conversion method is used and tracking is done in the whole process. Since the process needs to track the path so it also uses the Fuzzy Logic Controller to determine the position of the object and decline fake and unnecessary movements and track the right path [3].

Tracking can also be done by simple colour conversion technique that is conversion of RGB value to its corresponding HSB value. This is done by simple image processing techniques already present in the market. Blobs are also used as a way of calling the objects and images in a converted scenario of objects. This method is also simple the main work is done in background subtraction and then converting the colours of the images. The second work that is done is the fuzzy rules that need to be defined in such a way that it does not create a problem later on [4].

While researching over position tracking we also came across the system of DINDS. In this process the object to be tracked 
was observed by many cameras at a single time as the object moves from one place to another. The idea of taking so many cameras at one go was so that no single movement was missed by the system because that can cause error in tracking position. In such systems fuzzy logic is used in handing over the tracking ability from one camera embedded system to another. It gives an interesting technique of synchronisation of the system which there is a lot of movement going on [5].

\section{PROPOSED TECHNIQUE}

Various researches have been done in the field of position tracking. The technique proposed here firstly takes a video. The video can be of any object which is in motion but our system will only work in case of tracking of human motion. The whole process consists of four steps:
1) Identification of the object
2) Classification of the object
3) Tracking of object

\subsection{Identification Technique}

An image consists of a background as well as a foreground. For the precision in tracking, the object of interest has to be secluded from its background. There are various techniques using which the object can be isolated from its background but here in this paper the technique of background subtraction has been used. In this technique, a threshold value is set by image analysis. The intensities of pixels are calculated and a threshold value is set. If the current pixel value exceeds the value of the threshold then that pixel is considered as a foreground pixel else a background pixel which then set to gray level 0 .

\subsection{Classification Technique}

In this step of object tracking, machine learning has been used so as to differentiate between various objects of foreground. The process of machine learning has been used in the process as features are stored and based on that object of interest have been chosen. Machine learning can be of following types:
1) Supervised Learning
2) Unsupervised Learning
3) Reinforcement Learning

In supervised learning, a teacher like process is present which tells the result whereas in unsupervised learning the system learns by its experience. In reinforcement learning, there is nothing like teacher but a critic tells whether the output is correct or wrong. It does not give any other information about the result. For our system, supervised learning has been chosen.

The whole process of tracking can be represented by the flowchart in Fig 1.

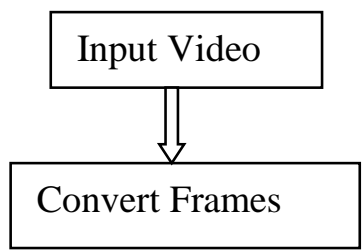

www.ijcat.com

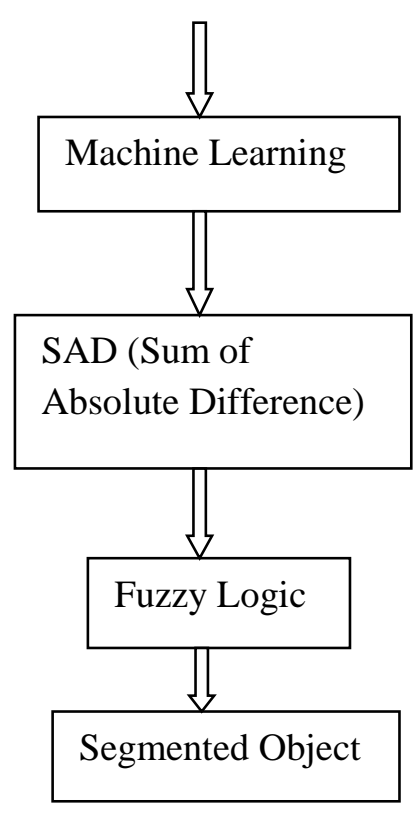

Fig 1.Flowchart showing Position Tracking.

\subsection{Tracking Technique}

At this level of process, the tracking of motion of a moving human object has been done. There are various techniques through which the motion of the object can be tracked. In the proposed system, the technique of SAD that is sum of absolute differences has been used. This calculates the interpixel difference between the two pixel locations of the two frames of same video. Then fuzzy rules are applied to the output which gives a fuzzy output which is converted into crisp value to detect the object motion.

\subsection{Algorithm For identification and Tracking Algorithm 1: Identification}

Fr_ $\_$bw = rgb2gray $(f r)$;

Fr_diff $=$ abs $($ double $(F r) b w)-$ double $($ bg_bw $)$;

If Fr_diff $>$ thresh pixel in foreground

For $\mathrm{j}=1$ : width

For k=1: height

If $(($ Fr_diff $(k, j)>$ thresh $))$

$$
\begin{aligned}
& f g(k, j)=F r \_b w(k, j) ; \\
& \text { else }
\end{aligned}
$$$$
\mathrm{fg}(\mathrm{k}, \mathrm{j})=0 \text {; }
$$ 
end

end

\section{Algorithm 2: Tracking}

$\mathrm{SAD}=\sum(\mathrm{X}-\mathrm{Y})$ for all pixels

Where $\mathrm{X}$ is the pixel of one frame and $\mathrm{Y}$ is the pixel of other frame.

\section{RESULTS}

Using the approach discussed above, the position of a lady in a video has been tracked. The following Fig. 2 depicts a scene in which a lady has been shown walking and the respective movement has been shown by applying the first step of the process to the image which is the technique of background subtraction. All the pixels which are in the background has been set to zero and only the object of interest that is a walking lady has been shown for tracking. The above explained scenario can be seen in the following image:

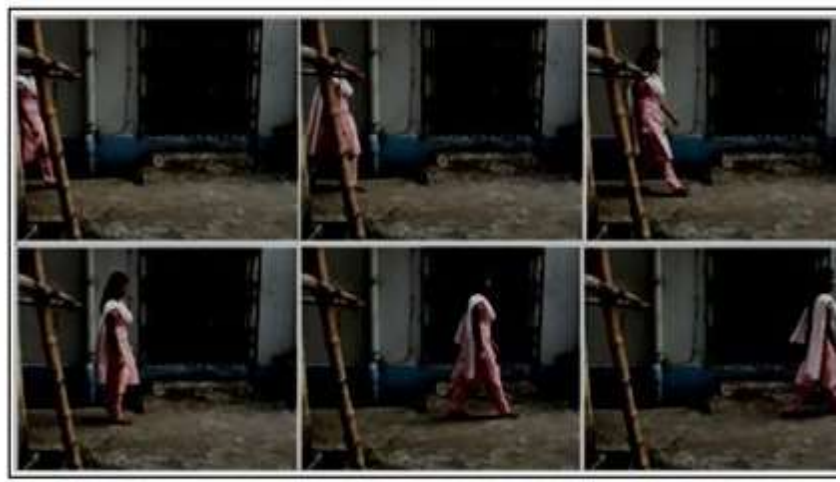

(a)

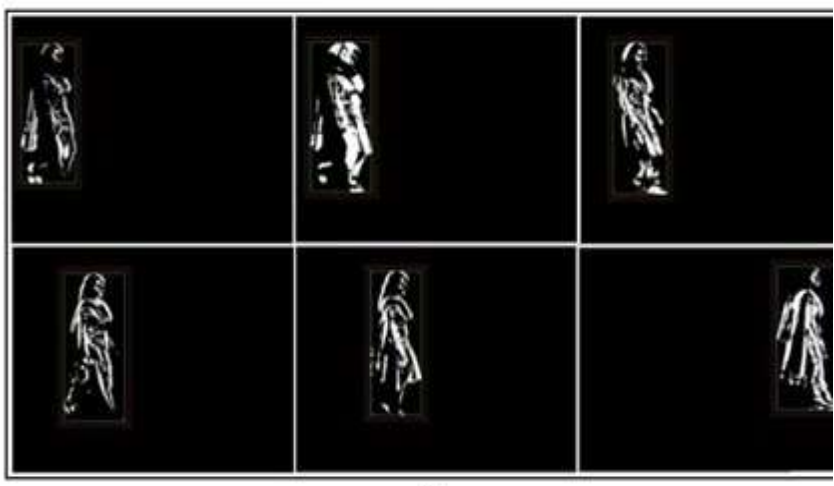

(b)

Fig 2. Frame Conversion and Background Subtraction

In the Fig.3, the technique of tracking described above has been applied to the two frames of an image and the resultant location with respect to time has been shown. The following image represents a graph showing the different movements of the lady with the values of $\mathrm{x}$ as well as $\mathrm{y}$ coordinates for four different scenarios. The above described algorithm has been used so as to obtain the results. This can be seen in the following figure:

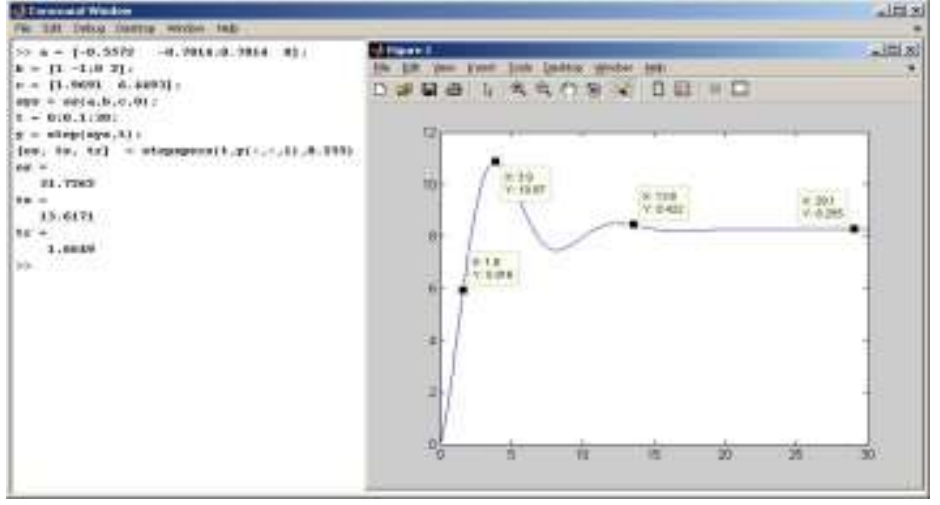

Fig 3.Tracked Path of the Lady.

\section{CONCLUSION}

This paper describes the process of Position Tracking using Fuzzy Logic by defining the various steps involved in doing this. This paper also gives the Algorithm used in this process. The algorithm developed clearly tells how the process is working and what all steps are used. The algorithm is divided into two parts identification and tracking. In this experiment we have used a video of a lady walking and its position is tracked.

\section{REFERENCES}

[1] Fuzzy Logic Path Planner and Motion Controller byEvolutionary Programming for Mobile Robots, Byung Cheol Min, Moon-Su Lee, and Donghan Kim.

[2] Neural Networks \& Fuzzy Logic Elakkiya Prabha T,PreFinalB.Tech-IT,M.Kumarasamy College Of Engineering, Karur Kiruthika M, Pre-Final,B.Tech-IT,M.Kumarasamy College Of Engineering, Karur

[3] Colour-Based Object Tracking and Following for Mobile Service Robots, Mohamed Abdellatif, Dept. of Mechatronics and Robotics., Egypt-Japan University of Science and Technology, Alexandria, EgyptTavel, P. 2007 Modeling and Simulation Design. AK Peters Ltd.

[4] Fuzzy Rule-based Classification of Human Tracking and Segmentation using Colour SpaceConversion, Sivabalakrishnan.M1 andDr.D.Manjula21Research Scholar, Department of CSE, College of Engineering, Anna University,Chennai, Tamil Nadu, India, sbkrishnanm@gmail.com, 2Assistant Professor, Department of CSE, College of Engineering, Anna University, Chennai, Tamil Nadu, India.

[5] ADAPTIVE CAMERA SELECTION BASED ON FUZZY AUTOMATON FOR OBJECT TRACKING IN A MULTICAMERA SYSTEM Kazuyuki MORIOKA1, Szilvester KOVÁCS2, Péter KORONDI3,Joo-Ho LEE4, Hideki HASHIMOTO5, 1Meiji University in Tokyo, JAPAN,2University of Miskolc, 3Budapest University of Technology and Economics, HUNGARY, 4Ritsumeikan University in Kyoto, 5University of Tokyo, JAPAN 